\title{
Prevalencia de los patrones electroforéticos de hemoglobina analizados en el CIHATA durante el período 2011-2013.
}

\author{
Solano-Vargas, Mariela., Salazar-Sánchez, Lizbeth., Buzano-Vargas, Lisa.
}

Centro de Investigación en Hematología y Trastornos Afines CIHATA, Universidad de Costa Rica

\section{RESUMEN}

Las hemoglobinopatías son alteraciones de las cadenas de globina que componen la hemoglobina, dentro de las alteraciones más comunes destacan las talasemias y la drepanocitosis. En el CIHATA se realizó un estudio retrospectivo de las muestras analizadas por electroforesis de hemoglobina durante el período 2011-2013, en donde se obtuvo un total de 2345 muestras analizadas, de las cuáles el patrón

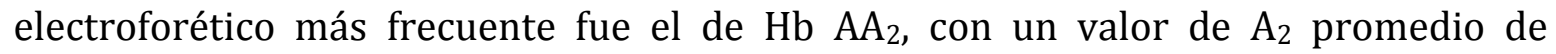
(4.8 \pm 0.8$) \%$, compatible con el diagnóstico de $\beta$ talasemia menor; seguido por el patrón asociado a portadores de $\mathrm{Hb} \mathrm{S}$. En menor cantidad se observaron resultados de $\mathrm{Hb} \mathrm{AC}, \mathrm{AF}, \mathrm{AA}_{2} \mathrm{~F}$, entre otros. Los patrones se distribuyeron a lo largo del país pero localizados mayormente en la región central (sesgo relacionado a mayor área de atracción). Es importante la detección de los patrones anormales, pues aunque no se asocien con un cuadro clínico, en condiciones de hipoxia o estrés fisiológico si se pueden presentar complicaciones graves y sobre todo para brindar consejo genético. Esta actualización brinda un panorama de la frecuencia de hemoglobinopatías circulantes en Costa Rica durante los últimos años. Se debe procurar la recopilación de información clínica, y demográfica completa de todos los pacientes, con el fin de hacer reportes de caso completos que puedan ayudar en el óptimo abordaje diagnóstico de los pacientes y familiares. 


\title{
Prevalence of hemoglobin electrophoretic patterns analyzed in CIHATA during 2011- 2013.
}

\begin{abstract}
Hemoglobinopathies are disorders of the globin chains that compose hemoglobin, among the most common alterations stand thalassemia and sickle-cell disease. In a retrospective study of the samples analyzed by hemoglobin electrophoresis at CIHATA for the period 2011-2013, where a total of 2345 analyzed samples were obtained, of which the most frequent electrophoretic pattern was the $\mathrm{Hb} \mathrm{AA}_{2}$, with a $\mathrm{A}_{2}$ average value of $(4.8 \pm 0.8) \%$, compatible with the diagnosis of $\beta$ thalassemia minor; followed by the associated pattern $\mathrm{Hb} \mathrm{S}$. Fewer results were observed with $\mathrm{Hb} \mathrm{AC}, \mathrm{AF}, \mathrm{AA}_{2} \mathrm{~F}$, among others. The patterns are distributed throughout the country but located mostly in the central region (area of greatest shipping samples to center). It is important to detect abnormal patterns, because although not associated with clinical, under hypoxic conditions or physiological stress serious complications can occur, and especially to provide genetic counseling. This update provides an overview of the frequency of circulating hemoglobin disorders in Costa Rica in recent years. Efforts should be made into collecting clinical information and complete clinical information of all patients, in order to complete case reports that can assist in the optimal diagnostic approach to patients and families.
\end{abstract}

\section{INTRODUCCIÓN}

La hemoglobina es una molécula formada por cuatro subunidades proteicas que se disponen de manera globular para almacenar al grupo hemo, este último en combinación con el 2,3 Difosfoglicerato, son responsables de la unión y afinidad del transporte de oxígeno hacia los tejidos. Es la naturaleza de las cadenas de globina lo que determina los diferentes tipos de hemoglobina que se forman normalmente en el adulto (Malcorra, 2001), tal como se muestra en el cuadro 1.
Cuadro 1. Tipo de hemoglobina, composición y valor de referencia normal para adultos utilizado en el CIHATA (Pañuela, 2009).

\begin{tabular}{lll}
\hline $\begin{array}{l}\text { Tipo } \\
\text { hemoglobina }\end{array}$ & $\begin{array}{l}\text { Globinas } \\
\text { presentes }\end{array}$ & $\begin{array}{l}\% \\
\text { normal } \\
\text { en adulto }\end{array}$ \\
\hline Hb A & $\alpha 2 \mathrm{y} \beta 2$ & $>90$ \\
Hb A2 & $\alpha 2$ y $\delta 2$ & $<3.8$ \\
Hb F & $\alpha 2$ y $\gamma 2$ & $<2.0$ \\
\hline
\end{tabular}

Los problemas en la formación y distribución de las cadenas de globinas se conocen como hemoglobinopatías, específicamente son alteraciones cualitativas $0 \quad$ cuantitativas consecuencia de mutaciones genéticas 
que modifican estructuralmente la proteína o que resultan en una disminución de la síntesis de las cadenas normales (Baeza, 2011). Pese a que algunas de estas hemoglobinopatías pueden cursar con cuadros clínicos severos, otras sólo presentarán patrones electroforéticos alterados sin clínica aparente.

Dentro de las hemoglobinopatías mayormente estudiadas se encuentran las talasemias (disminución de la síntesis de cadenas $\beta$ o $\alpha$ ), cuyo cuadro clínico es muy variado, incluso se ha tratado de denominar como síndrome talasémico por la hetorogeneidad de su sintomatología, no obstante, se resaltan características clásicos como anemia persistente y un extendido sanguíneo alterado con presencia de microcitos, hipocromía y con reporte hematimétrico de eritrocitosis (Saenz, 2006).

La drepanocitosis por su parte presenta un defecto estructural debido a la formación de hemoglobina $\mathrm{S}$, la cual posee menor afinidad por el oxígeno, ocasionando así rígidez y forma aberrante en los eritrocitos, lo que lleva a complicaciones de anemia grave y oclusiones vasculares (Svarch, 2009).

Otros patrones electroforéticos anormales se encuentran con presencia leve o casi nula de clínica asociada, por lo que pueden pasar desapercibidos durante la vida, y suelen tomar importancia cuando los pacientes se enfrenten a condiciones de estrés fisiológico como en el embarazo o cirugías.

\section{OBJETIVO}

Describir la frecuencia de los principales patrones electroforéticos analizadas en el CIHATA en el período 2011-2013.

\section{METODOLOGÍA}

Se realizó un estudio retrospectivo con base en las solicitudes de laboratorio enviadas por electroforesis de hemoglobina, con base en los datos demográficos y los resultados obtenidos durante los años 2011 al 2013.

Las muestras fueron procesadas mediante electroforesis en acetato de celulosa según Saenz et al, 2008. En caso de presentar un patrón compatible con aumento de $\mathrm{HbA}_{2} \mathrm{o} \mathrm{Hb}$ fetal estas se cuantificaron según los métodos de Marengo- Rowe (modificado por Alperin 1977) y Betke et al, respectivamente (Saenz G. , 2008).

\section{RESULTADOS}

Entre los años 2011 y 2013 se analizaron un total de 2345 muestras, provenientes de: región central, atlántica, pacifico central, zona norte y zona sur del país.

A continuación se muestra en la figura 1 , un resumen de los resultados obtenidos, destacando los principales patrones electroforéticos analizados.

Se obtuvo que la hemoglobinopatía más frecuente corresponde al patrón $\mathrm{Hb} \mathrm{AA}_{2}$ compatibles con beta talasemia menor, en donde el valor promedio de $\mathrm{Hb} \quad \mathrm{A}_{2}$ ronda en un $(4.8 \pm 0.8) \%$ aproximadamente con un $1 \%$ de incremento con respecto al valor de referencia normal. El segundo tipo de 
hemoglobinopatía encontrado fue el AS, en donde se tiene un alelo portador de la $\mathrm{Hb} \mathrm{S}$. Ambas alteraciones no se pueden asociar a una región geográfica en particular.

En menor medida se observan patrones de hemoglobinas anormales como AC, al igual que el AF compatible con $\alpha \delta$ talasemia y el $\mathrm{AA}_{2} / \mathrm{F}$ en donde se asocia a pacientes talasémicos con una compensación de $\mathrm{Hb}$ fetal debido a su su alta afinidad al oxígeno (Bradai, 2003).
Se detectó en el año 2012 un paciente con patrón SS, proveniente de la zona norte, pero del cuál no se tiene más información demográfica ni clínica.

\section{DISCUSIÓN}

Desde la década de los noventas el CIHATA ha recibido las electroforesis de hemoglobina de todas las regiones del país, en los últimos años los hospitales clase A realizan sus propias determinaciones, sin embargo el Centro sigue siendo un recinto importante de captación para las áreas de salud urbanas y rurales.

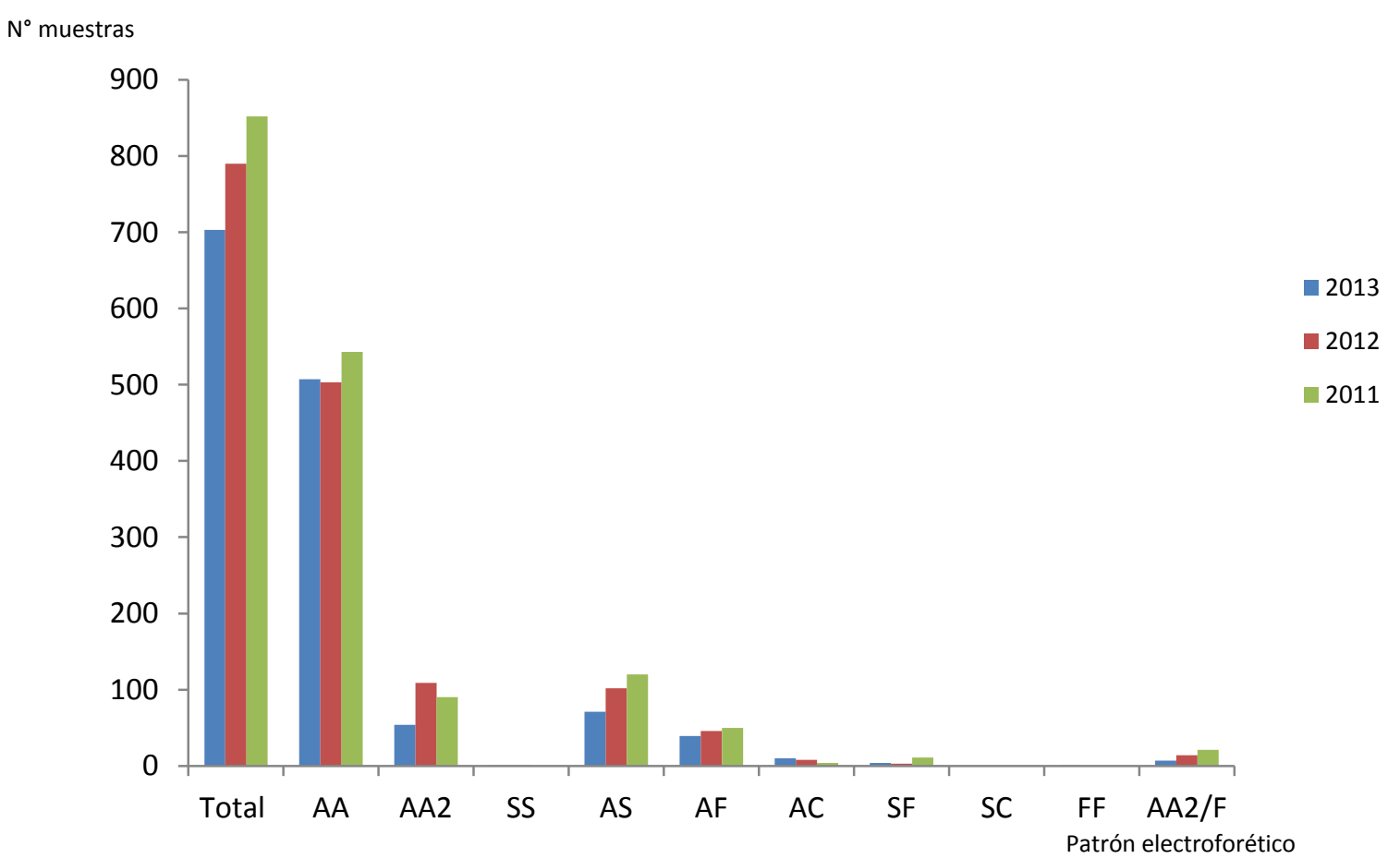

Figura 1. patrones electroforéticos obtenidos en el CIHATA, período 2011-2013 
De las 2345 muestras analizadas se encontró que los pacientes portadores de $\mathrm{Hb} \mathrm{AA}_{2}$ se encuentran distribuidos en todas las zonas del país en mayor o menor porcentaje, sin embargo esto tiene que ver con la cantidad de muestras enviadas por cada región, por tal motivo un $66 \%$ de los patrones $\mathrm{HBA}_{2}$ son provenientes de la región central, pese a esto, no se puede inferir en que los casos sean más frecuentes en esta zona con respecto a otras pues existe el sesgo de que la región central es el área con mayor captación de habitantes, y de centros de salud que refieren muestras al Centro.

Con respecto a los pacientes portadores del alelo de la $\mathrm{Hb}$ AS es importante su detección, ya que en la literatura se reporta una presencia escasa o nula de sintomatología, sin embargo en condiciones hipóxicas se han reportado complicaciones vasculares y trombóticas propias del síndrome drepanocítico; por tanto al reportar la alteración heterocigota a los pacientes y sus familiares se pretende que el personal médico tome medidas para brindar consejo genético que evite patrones homocigotos en su descendencia y un mejor abordaje del paciente en condiciones o estados de salud que así lo ameriten. (Saenz G. R., 2006).

La distribución de los alelos compatibles con talasemias menores y portadores drepanocíticos ha sido documentada en la población en años anteriores por Saénz et al, 1984 quienes reportan que la presencia del rasgo se atribuye a una distribución geográfica aleatoria debido a la dilución y entrecruzamiento étnico. Se debe tomar en cuenta que los estudios anteriores resultaron de muestreos aleatorios y en nuestro caso el estudio retrospectivo se basa en las muestras enviadas con sospecha clínica o historia familiar de hemoglobinopatía. (Saenz G. R., 2006).

\section{CONCLUSIÓN}

Es importante la constante actualización de los datos analizados en el centro, con el fin de detectar poblaciones en riesgo o cambios poblacionales importantes. El estudio de las hemoglobinopatías requiere de un abordaje integral, en donde se tomen en cuenta los valores hematimétricos, la morfología del glóbulo rojo, la cuantificación del hierro sérico, entre otros parámetros, los cuáles con el advenimiento de la tecnología son cada vez más sensibles. Tal es el caso del analizador de hemoglobina glicosilada que reporta algunas hemoglobinas anormales que deben ser confirmadas por electroforesis pero que son un filtro de detección más con el que se cuenta actualmente.

Esta revisión brinda un panorama actualizado de la frecuencia de hemoglobinas anormales circulantes en el país, se debe procurar la recopilación de información clínica, y demográfica completa de todos los pacientes, con el fin de hacer reportes de caso completos que puedan ayudar como herramienta diagnóstica óptima para el adecuado manejo de la población afectada. 


\section{BIBLIOGRAFÍA}

Baeza, A. (2011). Identificación de Síndromes talasémicos con base a la clasificación morfológica de anemias, recuento de reticulocitos y cuantificación de hierro sérico. México: Universidad Veracruzana.

Bradai, M. A. (2003). Hydroxyurea can eliminate transfusion requirment in chilren with severe B- thalassemia. Blood.

Malcorra, J. (2001). Hemoglobinopatías y talasemías. BSCP can Ped, 25(2), 265-277.

Pañuela, O. (2009). Recuperado el 7 de octubre de 2014, de http://colombiamedica.univalle. edu.co/index.php/comedica/art icle/view/366/1136

Saenz, G. (2008). Hematología Analítica. San José, Costa Rica: EDNASSS-CCSS.

Saenz, G. R. (2006). Síndromes talasémicos: Nuevos conceptos y estado actual del conocimiento en Costa Rica. Acta Médica Costarricense, 48(4).

Svarch, E. (2009). Fisiopatología de la drepanocitosis. Revista Cubana de Hematología e Inmunología, 25(1), 1-15. 